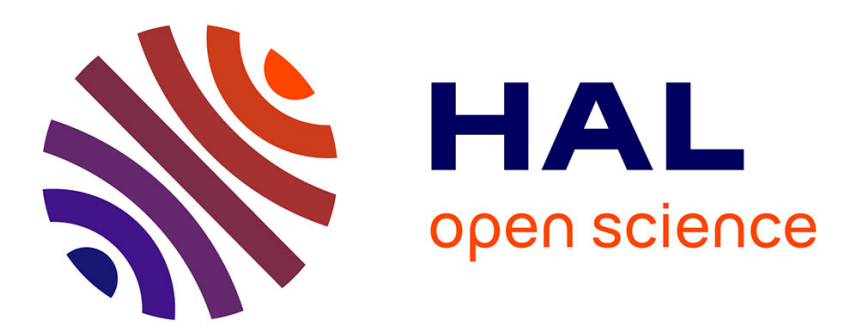

\title{
Caractérisation du fonctionnement physicochimique des sols forestiers par une approche expérimentale utilisant l'évolution de minéraux primaires
} Jacques Ranger, Michel Robert, Pascal Bonnaud, Michel Hervio, Jitka Lhomme

\section{To cite this version:}

Jacques Ranger, Michel Robert, Pascal Bonnaud, Michel Hervio, Jitka Lhomme. Caractérisation du fonctionnement physicochimique des sols forestiers par une approche expérimentale utilisant l'évolution de minéraux primaires. Agronomie, 1985, 5 (2), pp.157-168. hal-00884745

\section{HAL Id: hal-00884745 \\ https://hal.science/hal-00884745}

Submitted on 1 Jan 1985

HAL is a multi-disciplinary open access archive for the deposit and dissemination of scientific research documents, whether they are published or not. The documents may come from teaching and research institutions in France or abroad, or from public or private research centers.
L'archive ouverte pluridisciplinaire HAL, est destinée au dépôt et à la diffusion de documents scientifiques de niveau recherche, publiés ou non, émanant des établissements d'enseignement et de recherche français ou étrangers, des laboratoires publics ou privés. 


\title{
Caractérisation $\mathrm{du}$ fonctionnement physico- chimique des sols forestiers par une approche expérimentale utilisant l'évolution de minéraux primaires
}

\author{
Jacques RANGER \& Michel ROBERT (*) \\ avec la collaboration technique de Pascal BONNAUD, Michel HERVIO $\left({ }^{*}\right)$ \& Jitka LHOMME \\ I.N.R.A., Station de Recherches sur les Sols Forestiers et la Fertilisation, Centre de Recherches Forestières de \\ Nancy, Champenoux, F 54280 Seichamps \\ (*) I.N.R.A., Station de Science du Sol, route de St-Cyr, F 78000 Versailles
}

Ce travail s'inclut dans un programme général concernant l'effet des substitutions d'essence sur l'équilibre de l'écosystème forestier. Il concerne plus spécialement l'étude de l'évolution des sols par l'approche expérimentale dite des minéraux test : un minéral parfaitement connu, de bonne réactivité (ici un minéral complexe à base de mica, mica-chlorite et vermiculite) est utilisé pour identifier les processus pédogénétiques actuels en milieu acide.

Ces résultats montrent les premiers stades d'évolution du minéral après seulement 18 mois de contact dans les sols. Les éléments les plus accessibles (éléments échangeables et libres) ont subi des modifications notables. Les modifications des propriétés de surface du minéral se traduisent très nettement en minéralogie (diagrammes de diffraction $\mathrm{X}$ ). Les différents paramètres pris en compte (CEC, saturation cationique, éléments libres et minéralogie) donnent des réponses parfaitement cohérentes qui permettent de normaliser l'interprétation des résultats en identifiant les processus évolutifs. L'action des diverses essences forestières testées est prédominante dans les litières et les parties superficielles des horizons de surface $\left(\mathrm{A}_{11}\right)$. Plus profondément, c'est la constitution du milieu minéral qui joue le rôle fondamental sur l'orientation évolutive.

Parmi les essences, on note le comportement un peu particulier de l'épicéa : il produit, par exemple, toujours en surface la plus faible valeur de la CEC par rapport au groupe d'essences testées dans la même station. L'utilisation des minéraux test semble donc en adéquation avec la question que nous nous sommes posée même s'il est nécessaire de poursuivre plus longtemps l'expérience pour qu'une bonne discrimination des espèces et des milieux s'opère.

Mots clés additionnels : Altération expérimentale, vermiculite, test minéral, essences forestières, mica interstratifié, chlorite. in situ evolution of primary minerals.

This study was part of a general programme, concerning the change in ecosystem equilibrium when a new forest species has been introduced. It concerned more specifically the evolution of soil as studied by an experimental approach, called the mineral weathering test method. A well known mineral with high reactivity - in this case a vermiculite (whith some mica, and interstratified mica-chlorite) - was used to identify present pedogenetic processes in acidic soils. Resuits showed the first stages of mineral evolution, after only 18 months of contact with soil. The more accessible elements such as exchangeable ions and free oxides underwent important changes. X-ray diffraction studies clearly showed the result of changes in the mineral's surface properties. The different parameters such as CEC exchangeable ions, free oxides and mineralogy indicated similar evolutionary tendencies which allowed us to identify pedogenetic processes. In litter and $A_{11}$ horizon samples, the influence of forest species was dominant but below the $A_{12}$ horizon the influence of the mineral soil became more important. Among the species we was tested, spruce showed particular behaviour, resulting in divergent evolution of mineral test properties from other species. The mineral test method seemed adequate for answering the question we was posed, even if it was necessary to continue experiments longer, in order to obtain. better differentiation between species and soils.

Additional key words : Experimental weathering, vermiculite, test mineral, forest species, micachlorite interstratified mineral. 


\section{INTRODUCTION}

Lorsque l'on substitue une essence allochtone, le plus souvent résineuse et cultivée en monoculture, à la forêt climacique, on modifie le fonctionnement de l'écosystème. Des études ont été menées, en France et à l'étranger, pour mesurer l'amplitude des transformations induites par diverses essences et pour différentes conditions de sol. Ces études ont porté soit uniquement sur le sol (BONNEAU et al., 1977; NYS, $1981 a$ et $b$; HeRBAULTS \& DEBUYLE, 1981), soit plus globalement sur le fonctionnement du système (NOIRFALISE \& VANESSE, 1975 ; CR-ASP PIREN, 1982 ; NYS et al., 1983).

Les 2 aspects, évolution pédogénétique et maintien de la fertilité des sols, sont fondamentaux. A cet égard, les études effectuées par la méthode des bilans de matière sont une approche séduisante puisqu'elles permettent de comparer, toutes choses égales par ailleurs, l'effet de plusieurs essences sur un sol donné. De fait, ce type d'approche n'est pas sans difficultés : en effet,

a) les situations parfaitement comparables sont très rares,

b) les modifications attribuables à la seule substitution d'essence sont souvent très tenues et elles se diluent dans le cumul des évolutions passées et actuelles ; leur observation nécessite une approche parfaitement rigoureuse et précise incompatible avec toute hétérogénéité,

c) ces études sont très lourdes av plan analytique excluant l'analyse d'un grand nombre de situations.

Ces réflexions nous ont conduits à adopter une démarche nouvelle utilisée par BERTHELIN et al. (1983) qui consiste globalement à rajeunir le système minéral du sol que l'on veut étudier. La méthode consiste à inclure dans les sols, des minéraux purs qui représentent les grandes familles des silicates des sols (tecto-silicates, phyllosilicates). Plusieurs séries sont installées, permettant de suivre après différentes durées de séjour, l'évolution du minéral que l'on comparera au minéral initial.

Elle permet de s'affranchir partiellement des problèmes d'hétérogénéité des sols et donc de comparer de nombreuses situations et d'aborder le problème de dynamique d'évolution. Il ne s'agit cependant que d'un test qui permet de comparer entre elles des situations. Il reste bien évidemment très difficile d'aborder par cette méthode les vitesses de réaction des minéraux des sols. Il faut noter enfin que cette approche fait partie d'une stratégie expérimentale qui n'exclut pas pour autant la poursuite de l'étude des sols en place.

\section{MATÉRIEL ET MÉTHODES}

Dans un premier temps, nous avons retenu d'expérimenter in situ avec un seul minéral test. Il s'agit alors de choisir une composition minéralogique adaptée aux problèmes étudiés (bonne réactivité) et, de plus, d'introduire ce matériel dans le milieu naturel en le perturbant le moins possible. Enfin, il s'agit de pouvoir suivre et caractériser les évolutions subies. Pour cela, un certain nombre d'opérations sont nécessaires :

\section{A. Caractérisation et préparation du minéral test}

Un minéral nous a été fourni par la Société ELFISOLATION, sous le nom de "vermiculite du Kenya ». Il s'agit en fait, comme dans la majorité des cas pour ces vermiculites commerciales, d'un minéral complexe dont le comportement global indique que l'on a affaire à l'association de 3 types de feuillets : des feuillets de type mica, des feuillets de type chlorite et des feuillets de type vermiculite. On est en présence de séquences monominérales - mica et vermiculite et de séquences interstratifiées régulières de micachlorite. La valeur de la capacité d'échange du matériau (31 meq pour $100 \mathrm{~g}$ ) indique que la vermiculite n'est pas majoritaire dans ce minéral. Ce premier inconvénient s'est révélé largement compensé par l'existence au sein d'une même particule (de taille sableuse 50 à $100 \mu \mathrm{m}$ ) de 3 minéraux qui constituent de véritables références « internes » pour la diffraction des rayons $\mathrm{X}$, rendant ainsi possible la caractérisation d'une évolution relative des constituants.

Ce minéral a une granulométrie initiale de 1 à $2 \mathrm{~cm}$. Celle-ci est ramenée, par broyage (le moins énergique possible) et tamisage successifs (dont les derniers sont effectués par voie humide), à une dimension de particules comprise entre 50 et $100 \mu \mathrm{m}$. Le broyage n'imprime pas de modification apparente au niveau des feuillets (jugée par diffraction RX), mais par contre, il peut changer la morphologie des particules (rebroussements des bords des particules de 50 à $100 \mu \mathrm{m})$. La référence expérimentale est le minéral broyé et $3 \mathrm{~g}$ de ce minéral sont enfermés dans un sachet polyamide imputrescible (NYTREL TI) de $10 \times 5 \mathrm{~cm}$ dont la maille de la toile est de $20 \mu \mathrm{m}$.

Remarque: L'approche expérimentale que nous avons adoptée présente des avantages considérables par rapport aux études de sol en place : la référence expérimentale est parfaitement fixée et la réactivité d'un minéral non altéré assure une évolution rapide.

Elle présente cependant des inconvénients qu'il est nécessaire de souligner, mais qui ne nous ont pas paru rédhibitoires : malgré toutes les précautions que l'on peut prendre, la perturbation du milieu existe; le minéral est isolé d'une partie des agents biologiques intervenant dans l'évolution du sol et se trouve localement beaucoup plus concentré qu'il ne le serait dans le sol en place ; enfin, il est toujours possible de placer le minéral en condition particulière, notamment dans les sols caillouteux.

\section{B. Positionnement du minéral dans le site d'expéri- mentation}

Ces sachets sont placés dans le sol à partir du front d'une fosse pédologique, à la base des horizons suivants : litière, $A_{11}(-5 \mathrm{~cm}), A_{12}(-15 \mathrm{~cm})$. Ces fosses sont creusées perpendiculairement à la pente du terrain pour éviter de trop modifier les conditions hydrologiques au niveau des échantillons introduits. Ces fosses sont rebouchées en respectant la différenciation des horizons pédologiques.

\section{Les stations expérimentales}

Elles sont présentées très globalement dans le tableau 1. Nous avons choisi des stations déjà étudiées par NYS $(1975 ; 1981 a$ et $b)$ en ce qui concerne les 
TABLEAU 1

Quelques caractéristiques générales des stations expérimentales. Some general characteristics for experimental sites.

\begin{tabular}{|c|c|c|c|c|c|c|c|c|c|c|}
\hline Localisation & $\begin{array}{l}\text { Climat gén } \\
\text { Temp. moy. } \\
\text { annuelle }\end{array}$ & $\begin{array}{l}\text { néral } \\
\text { Pluvio. } \\
\text { mm }\end{array}$ & Altitude & R.M. & Type & Humus & $\begin{array}{l}\text { Sol } \\
\qquad \begin{array}{c}\mathrm{pH} \\
\left(\mathrm{H}_{2} \mathrm{O}\right)\end{array}\end{array}$ & $\begin{array}{c}\text { Taux } \\
\text { d'argile }\end{array}$ & $\begin{array}{c}\text { Essences } \\
\text { comparées }\end{array}$ & Référence \\
\hline $\begin{array}{l}\text { Ardennes primaires } \\
\text { Forêt de Château } \\
\text { Regnault }\end{array}$ & $8^{\circ} \mathrm{C}$ & 1100 & $390 \mathrm{~m}$ & $\begin{array}{l}\text { Altérite limoneuse } \\
\text { de schistes } \\
\text { Reviniens }\end{array}$ & $\begin{array}{l}\text { Brun } \\
\text { acide }\end{array}$ & $\begin{array}{l}\text { Mull } \\
\text { acide } \\
\text { à Moder }\end{array}$ & 4,0 & $13 \%$ & $\begin{array}{l}\text { Chêne, Hêtre } \\
\text { Epicea }\end{array}$ & $\begin{array}{c}\text { NYS } \\
(1981 b)\end{array}$ \\
\hline $\begin{array}{l}\text { Normandie } \\
\text { Basse Forêt d'Eu }\end{array}$ & $10^{\circ} \mathrm{C}$ & 1100 & $200 \mathrm{~m}$ & $\begin{array}{l}\text { Limons éolien } \\
\text { sur argile } \\
\text { à silex }\end{array}$ & $\begin{array}{l}\text { Brun } \\
\text { acide }\end{array}$ & $\begin{array}{l}\text { Mull } \\
\text { acide } \\
\text { à Moder }\end{array}$ & $\begin{array}{c}4,0 \\
\grave{a} \\
4,5\end{array}$ & $20 \%$ & $\begin{array}{l}\text { Epicea, Douglas, } \\
\text { Mélèze, Hêtre }\end{array}$ & $\begin{array}{c}\text { NYS } \\
(1981 a)\end{array}$ \\
\hline $\begin{array}{l}\text { Massif Central } \\
\text { Plateau } \\
\text { de Millevaches } \\
\text { Peyrelevade }\end{array}$ & $8^{\circ} \mathrm{C}$ & 1400 & $950 \mathrm{~m}$ & $\begin{array}{l}\text { Arène de granite } \\
\text { à } 2 \text { micas }\end{array}$ & $\begin{array}{l}\text { Podzol } \\
\text { humi- } \\
\text { fère }\end{array}$ & $\begin{array}{l}\text { Moder } \\
\text { à Mor }\end{array}$ & 4,0 & $10 \%$ & $\begin{array}{l}\text { Epicea, Lande } \\
\text { à Callune }\end{array}$ & $\begin{array}{l}\text { NyS } \\
(1975)\end{array}$ \\
\hline $\begin{array}{l}\text { Bassin parisien } \\
\text { Cires-lès-Mello }\end{array}$ & $10^{\circ} \mathrm{C}$ & 600 & $100 \mathrm{~m}$ & $\begin{array}{l}\text { Sables de Beau- } \\
\text { champ (quartzo- } \\
\text { feldspaths) } \\
\text { Bartonien }\end{array}$ & Podzol & Mor & 4,0 & $5 \%$ & $\begin{array}{l}\text { Mélange résineux } \\
\text { (Pin sylv. Epicea) } \\
\text { Mélange feuillu } \\
\text { (Hêtre, Chêne) }\end{array}$ & $\begin{array}{l}\text { NYS } \\
(1981 a)\end{array}$ \\
\hline
\end{tabular}

bilans de matière. Ceci nous permettra ainsi de confronter les 2 approches, mais aussi de formuler un diagnostic mieux argumenté sur l'influence des essences dans ces stations.

Cette $1^{\text {re }}$ expérience ne concerne que 4 sites, volontairement limités au milieu acide et couvrant une gamme de fertilité de station allant des sols très pauvres (podzols de Cires-lès-Mello) aux sols limoneux pauvres (sol brun acide des Ardennes) et plus riches (sol brun acide de Normandie). Le cas du sol organique de Peyrelevade est particulier.

\section{Suivi des évolutions du minéral test : analyses et types de caractérisation réalisés}

Après avoir été retirés du sol, les minéraux font l'objet des analyses suivantes :

- observation au microscope optique et électronique à balayage à l'état humide (JEOL 35 avec microsonde TRACOR couplée),

- analyse chimique totale par fusion au métaborate de strontium : méthode JEANROY (1974),

- extraction des éléments « libres »: méthode MEHRA-JACKSON (1960),

- analyse physico-chimique : capacité totale d'échange (CEC).

Celle-ci est définie comme la somme des cations échangeables déplacés par $\mathrm{KCl}$ normal pour doser $\mathrm{Ca}$, $\mathrm{Mg}, \mathrm{Na}, \mathrm{Mn}$, Fe et par $\mathrm{HN}_{4} \mathrm{Cl} \mathrm{N}$ pour doser $\mathrm{K}$, augmentée des cations acides ( $\mathrm{Al}$ et $\mathrm{H})$. Les éléments $\mathrm{Ca}$, $\mathrm{Mg}, \mathrm{Na}, \mathrm{Mn}, \mathrm{Fe}, \mathrm{Al}$ sont dosés par spectrophotométrie d'absorption atomique, $\mathrm{K}$ par spectrophotométrie d'émission atomique; l'acidité totale d'échange qui est la somme des protons et de l'aluminium échangeable est définie par titrage potentiométrique jusqu'à pH 8,4. Les protons échangeables sont évalués par différence entre acidité d'échange et aluminium échangeable (ROUILLER et al., 1980).

- Analyse minéralogique en diffraction des rayons $X$ (appareil Philips avec anticathode au cobalt) sur des échantillons "bruts " (saturation issue du contact avec le sol), puis après saturation au $\mathrm{KCl} \mathrm{N}$ et chauffage progressif, ou sur des échantillons après extraction des éléments "libres » et saturation potassique.

- Des analyses thermo-pondérale et thermique différentielle si nécessaire.

Les résultats présentés ici correspondent à des niveaux installés en octobre 1979 et retirés en avril 1981.

\section{RÉSULTATS ET INTERPRÉTATIONS}

\section{A. Les observations}

Au microscope à balayage, où l'on peut observer les particules de taille sableuse comprenant l'ensemble des minéraux déterminés aux rayons $\mathrm{X}$, les différences avec le minéral témoin sont faibles voire inexistantes. La pression du sol a cependant conduit à une orientation préférentielle des phyllosilicates. On peut de même observer une pénétration des racines fines et du mycélium dans les sachets. Dans un cas particulier (litière de Douglas, Normandie), on constate une imprégnation de manganèse visible au microscope à balayage et décelée à la microsonde ; c'est le seul cas où une structuration de l'échantillon en micro-agrégat est apparue.

\section{B. Les analyses totales}

L'analyse totale effectuée sur le minéral de référence donne les résultats suivants ;

$\begin{array}{rccccc}\mathrm{SiO}_{2} & \mathrm{Al}_{2} \mathrm{O}_{3} & \mathrm{Fe}_{2} \mathrm{O}_{3} & \mathrm{CaO} & \mathrm{K}_{2} \mathrm{O} & \mathrm{MgO} \\ 40,25 & 10,80 & 5,90 & 0,41 & 5,76 & 29,35 \\ \mathrm{MnO} & \mathrm{Na}_{2} \mathrm{O} & \mathrm{TiO}_{2} & \mathrm{H}_{2} \mathrm{O}^{+} & \Sigma & \\ 0,03 & 0 & 0,67 & 9,1 & 102,3 & \end{array}$

Les variations observées, en moyenne assez faibles, portent sur la perte au feu et les autres éléments constitutifs des silicates. 


\section{La perte au feu}

Elle augmente le plus souvent, traduisant partiellement la variation de l'hydratation des feuillets et/ou une certaine fixation de matière organique (fig. 1).

L'hydratation est le $1^{\text {er }}$ processus de déstabilisation du minéral, qui provoque une ouverture des feuillets de type mica, ou de l'interstratifié. Cette fixation de molécules d'eau coïncide avec l'augmentation de la CEC : de fait, il existe une relation globale significative à 5 p. $100(r=0,389 n=33)$ entre perte au feu et CEC du minéral. En réalité, les phénomènes sont plus complexes dans la mesure où la matière organique fixée joue un rôle et où tous les horizons ne se comportent pas de la même façon ; ainsi, les relations statistiques partielles pour chaque horizon sont d'autant meilleures que l'on se rapproche de la surface du sol, là justement où l'augmentation de la CEC est la plus forte (déstabilisation de l'interstratifié).

\section{Les constituants élémentaires des silicates}

Si l'on compare les résultats directs de l'analyse ou les rapports moléculaires, les évolutions sont peu marquées. Les modifications portent d'abord sur les éléments facilement accessibles qui n'ont pas le plus souvent un poids important dans l'analyse totale. On constate ainsi des pertes légères en $\mathrm{CaO}$ et $\mathrm{MgO}$ et une accumulation relative de $\mathrm{Al}_{2} \mathrm{O}_{3}$ et $\mathrm{MnO}$.

On observe en fait la résultante de 2 processus :

- le minéral placé dans des conditions thermodynamiques totalement différentes de celles correspondant

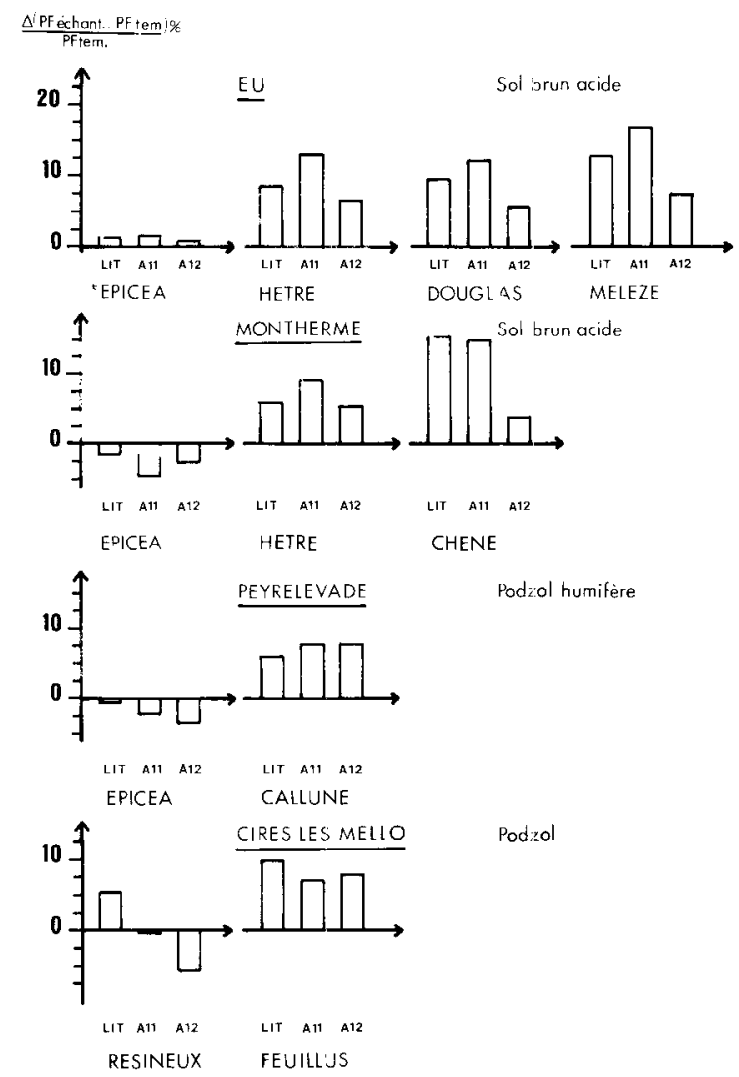

Figure 1

Perte au feu à $1000{ }^{\circ} \mathrm{C}$ (variation en pourcentage par rapport au témoin).

Residual humiditv at I $000{ }^{\circ} \mathrm{C}$ (variation relative to control). à sa formation subit une déstabilisation, en milieu lessivant, sous l'influence des eaux de drainage chargées en acides organiques et minéraux dilués ;

- le minéral échange et/ou fixe des éléments provenant de la minéralisation des litières constamment apportés à la surface du sol par le cycle biologique (sels d'acides organiques et minéraux). Ce processus s'oppose donc en partie au précédent : le cas du manganèse est typique à cet égard.

L'analyse chimique totale ne permet pas actuellement une bonne discrimination des échantillons. Seule la perte au feu permet une certaine hiérarchisation. En effet, il ressort de la figure 1 que le comportement de l'épicéa (ainsi que celui des résineux de Cires-lèsMello) est caractéristique ; la perte au feu des minéraux des pessières est systématiquement plus faible que celle des minéraux issus des autres peuplements d'un même site. Dans l'absolu, elle est parfois légèrement supérieure, mais le plus souvent inférieure à celle du témoin. Pour ce critère, l'épicéa a tendance à homogénéiser l'effet des milieux, dans la mesure où les échantillons des pessières du sol brun acide des Ardennes et du podzol de Peyrelevade ont par exemple le même comportement. Ce comportement spécifique ne semble donc pas fortuit sans que, pour l'instant, on puisse donner d'explication définitive. La valeur systématiquement plus faible du rapport moléculaire $\frac{\mathrm{SiO}_{2} \text { total }}{\mathrm{K}_{2} \mathrm{O} \text { total }}$ en litière et $\mathrm{A}_{11}$, pour l'épicéa dans chaque station (sans que le potassium échangeable soit affecté), fait penser à une tendance à la rétrogradation potassique bloquant la $\mathrm{CEC}$. Il pourrait s'agir alors d'une conséquence d'un recyclage biologique plus efficace de cet élément par cette espèce. L'étude en cours sur le même minéral ayant séjourné 3 ans dans les sols devrait nous permettre de préciser ce comportement.

\section{Capacité totale d'échange et saturation cationique}

Ces propriétés ont été fortement modifiées après une période limitée à 18 mois de contact dans le sol. Globalement la CEC a varié dans une fourchette atteignant $\pm 30 \mathrm{p}$. cent de sa valeur initiale.

Nous avons souligné la relation globale qui existe entre la perte au feu du minéral et la variation de CEC traduisant une certaine ouverture des feuillets. Si l'on se réfère à la figure 2 , on peut dégager plusieurs grandes tendances évolutives :

- Le critère profondeur de prélèvement marque assez fortement l'évolution de la CEC. On constate qu'il existe une liaison statistique globale entre la CEC et la profondeur du prélèvement $(\mathrm{n}=33 \mathrm{r}=0,603)$. Cela traduit le fait que la CEC augmente (comparativement au témoin) pour les minéraux placés sous les litières, alors que la tendance est inverse pour les minéraux placés dans les sols.

- Si on considère les échantillons placés sous les litières et à la base de l'horizon $\mathrm{A}_{11}(-5 \mathrm{~cm})$, une espèce, l'épicéa, se distingue assez nettement ; elle se caractérise toujours par la CEC la plus faible relativement au groupe d'espèces présent dans une station donnée. Le milieu imprime en outre un effet propre.

- En ce qui concerne les échantillons placés sous l'horizon $A_{12}(-15 \mathrm{~cm})$, l'effet essence ne ressort que faiblement; par contre, la discrimination liée au 

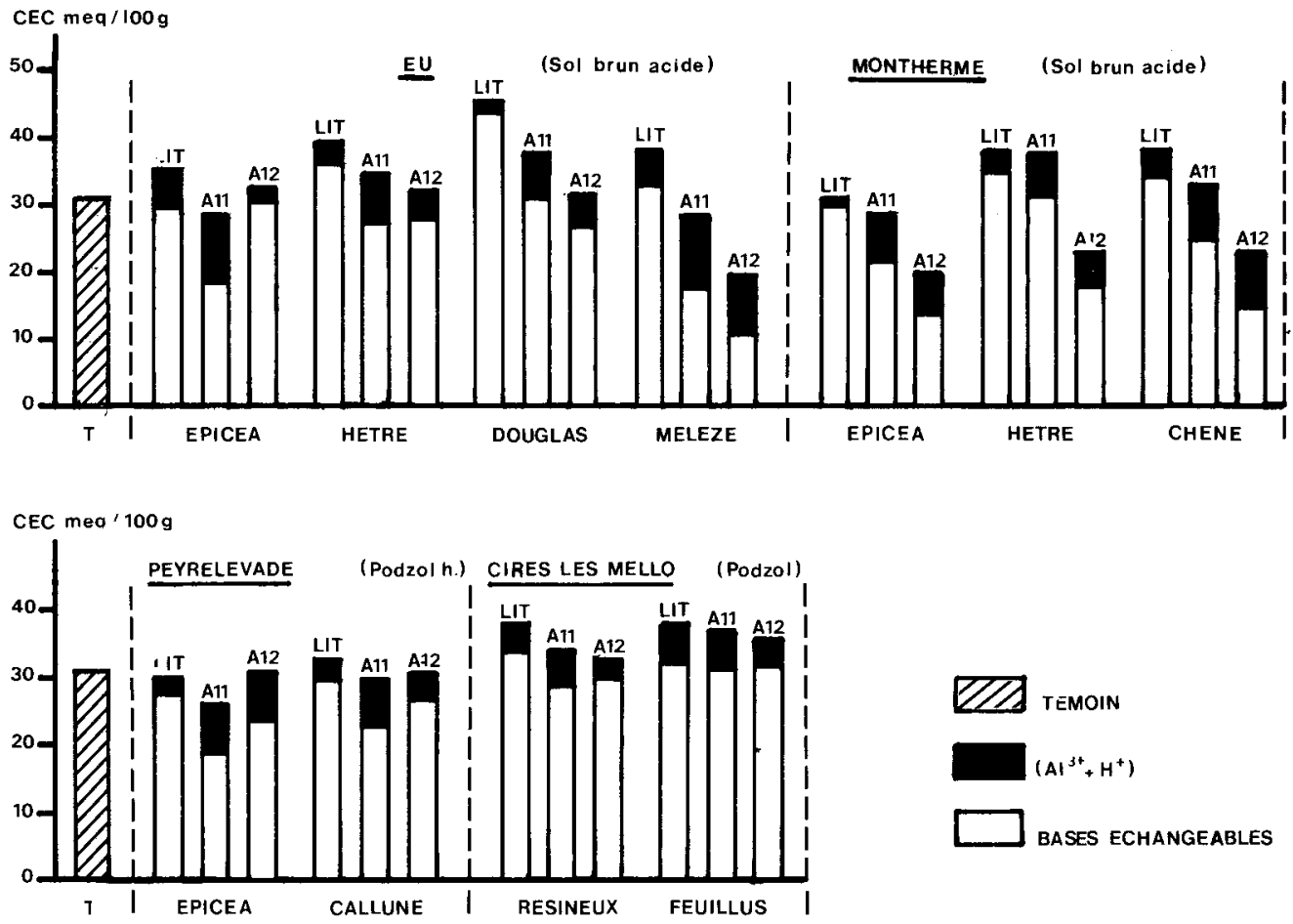

Figure 2

Premiers stades d'évolution in situ des minéraux test : capacité d'échange.

milieu devient très apparente. Les 4 stations étudiées sont bien séparées, les critères espèces ne provoquant pas globalement de dispersion majeure à l'intérieur d'une station (sauf dans le cas des sols de la forêt d'Eu).

En ce qui concerne la saturation cationique, le tableau 2 révèle les tendances observées sur les 3 cations dominants $(\mathrm{Al}, \mathrm{Mg}$ et $\mathrm{Ca})$ :

- Les tendances dans leurs distributions verticales montrent une certaine opposition entre les échantillons placés sous les litières et ceux provenant des sols, le $\mathrm{Mg}$ et le $\mathrm{Ca}$ étant globalement plus abondants sous litières que dans les sols, alors que l'inverse est vrai pour l'Al.

- Des relations statistiques intéressantes existent entre les cations saturant l'argile. $\mathrm{Mg}$ et $\mathrm{Al}$ sont reliés négativement $(\mathrm{r}=-0,580 \mathrm{n}=33)$ ainsi que $\mathrm{Ca}$ et Al $(r=-0,327 n=33)$.

La $1^{\text {re }}$ relation, $(\mathrm{Mg}$ éch. $)=\mathrm{f}(\mathrm{Al}$ éch.) (fig. 3$)$, montre l'antagonisme entre ces cations, dont la présence en zone interfoliaire traduit 2 processus divergents (ROBERT et al., 1979). Le maintien du Mg sur la CEC, dans la zone de $\mathrm{pH}$ considérée, traduit, pour le minéral utilisé très riche en $\mathrm{Mg}$, une altération en milieu acide et complexant : il s'agit des minéraux placés sous les litières dans toutes les stations, mais aussi des minéraux issus des sols podzolisés (Cires-lès-Mello et Peyrelevade, sans distinction d'essence et d'horizon) ; à l'opposé, quand l'acidité domine (c'est le cas des horizons profonds), le $\mathrm{Mg}$ est fortement lessivé. La présence d'Al échangeable traduit plutôt une dynamique acide non complexante qui est essentiellement le fait des horizons hémiorganiques des sols; là encore, on retrouve le comportement parallèle des litières et des minéraux issus de tous les horizons des
First steps of evolution for the primary minerals left 18 months in situ : cation exchange capacity.

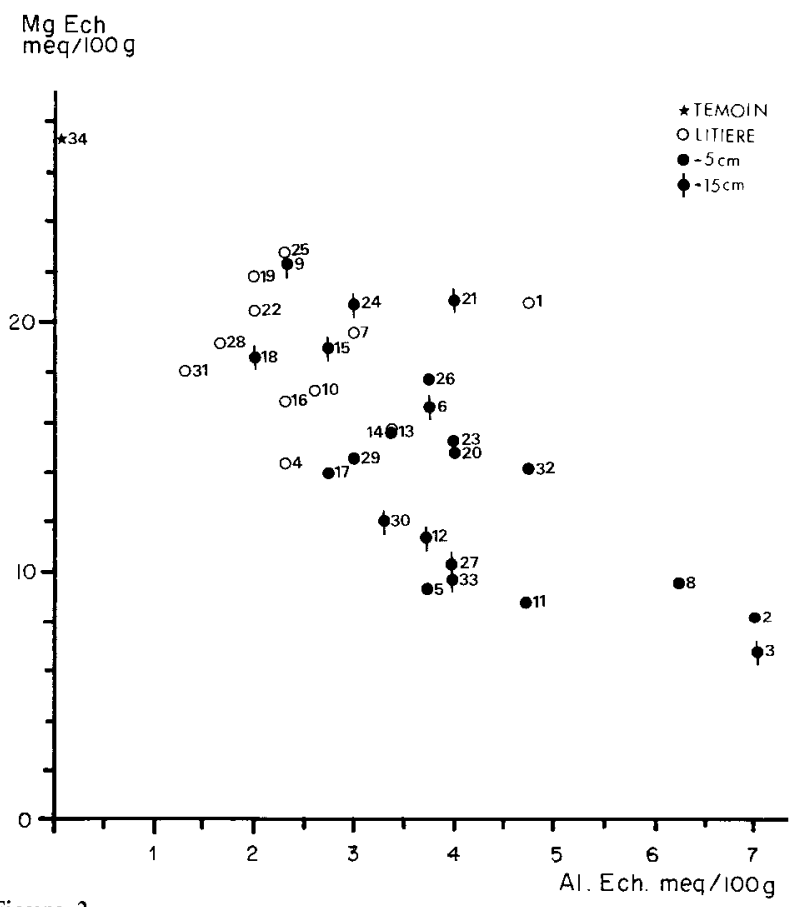

Figure 3

Liaison entre magnésium et aluminium échangeables.

Interrelation between exchangeable magnesium and exchangeable aluminium.

podzols. Il n'existe pas de différence entre les essences d'une même station.

La relation négative trouvée entre le $\mathrm{Ca}$ et l'Al échangeables traduit globalement l'opposition entre les comportements des minéraux issus des litières et ceux issus des horizons hémiorganiques. Elle relate bien à cet égard la résultante de processus divergents. 
TABLEAU 2

Les éléments échangeables (meq pour $100 \mathrm{~g}$ de minéral séché à l'air). Exchangeable cations (meq per $100 \mathrm{~g}$ of air dried mineral).

\begin{tabular}{|c|c|c|c|c|c|c|c|c|c|c|c|c|c|}
\hline Site & Essence & Horizon & $\begin{array}{l}\mathrm{n}^{\circ} \\
\text { ordre }\end{array}$ & $\begin{array}{c}\text { Acidité } \\
\mathrm{H}+\mathrm{Al}^{3+}\end{array}$ & $\mathrm{Al}$ & $\mathrm{Na}$ & $\mathrm{Ca}$ & $\mathrm{Mn}$ & $\mathrm{Fe}$ & $\mathbf{K}$ & $\mathrm{Mg}$ & $S$ & Total \\
\hline \multirow[t]{11}{*}{$\mathrm{Eu}$} & \multirow[t]{2}{*}{ Mélèze } & $\begin{array}{l}\text { Lit. } \\
-5\end{array}$ & $\begin{array}{l}1 \\
2\end{array}$ & $\begin{array}{r}5,6 \\
10,9\end{array}$ & $\begin{array}{l}4,7 \\
7,0\end{array}$ & $\begin{array}{l}1,2 \\
1,0\end{array}$ & $\begin{array}{l}8,6 \\
6,3\end{array}$ & $\begin{array}{l}1,1 \\
1,0\end{array}$ & $\begin{array}{l}0,2 \\
0,3\end{array}$ & $\begin{array}{l}0,8 \\
0,8\end{array}$ & $\begin{array}{r}20,8 \\
8,1\end{array}$ & $\begin{array}{l}32,7 \\
17,5\end{array}$ & $\begin{array}{l}38,3 \\
28,4\end{array}$ \\
\hline & & -15 & 3 & 9,4 & 7,0 & 1,2 & 1,4 & 0,4 & 0,3 & 0,7 & 6,3 & 10,3 & $\begin{array}{l}20,4 \\
19,7\end{array}$ \\
\hline & \multirow[t]{3}{*}{ Douglas } & Lit. & 4 & 2,0 & 2,3 & 2,6 & 24,0 & 2,3 & 0,0 & 0,6 & 14,0 & 43,5 & 45,5 \\
\hline & & -5 & 5 & 6,4 & 3,7 & 2,2 & 16,5 & 1,8 & 0,3 & 0,6 & 9,5 & 30,9 & 37,3 \\
\hline & & -15 & 6 & 5,0 & 3,7 & 1,3 & 6,7 & 1,1 & 0,2 & 0,7 & 16,3 & 26,3 & 31,3 \\
\hline & \multirow[t]{3}{*}{ Epicéa } & Lit. & 7 & 5,1 & 3,0 & 1,2 & 7,3 & 0,9 & 0,2 & 0,6 & 19,6 & 29,8 & 34,9 \\
\hline & & -5 & 8 & 10,3 & 6,3 & 1,6 & 5,1 & 0,6 & 0,3 & 0,7 & 9,9 & 18,2 & 28,5 \\
\hline & & -15 & 9 & 1,9 & 2,3 & 1,3 & 5,4 & 0,7 & 0,0 & 0,8 & 22,2 & 30,4 & 32,3 \\
\hline & \multirow[t]{3}{*}{ Hêtre } & Lit. & 10 & 3,7 & 2,7 & 1,4 & 15,0 & 1,6 & 0,0 & 0,6 & 17,4 & 36,0 & 39,7 \\
\hline & & -5 & 11 & 7,8 & 4,7 & 1,6 & 14,8 & 0,8 & 0,3 & 0,8 & 8,8 & 27,1 & 34,9 \\
\hline & & -15 & 12 & 4,9 & 3,7 & 1,6 & 12,2 & 1,0 & 0,2 & 0,8 & 11,6 & 27,4 & 32,3 \\
\hline \multirow[t]{6}{*}{ Cires-lès-Mello } & \multirow[t]{3}{*}{ Feuillus } & Lit. & 13 & 5,3 & 3,3 & 1,0 & 13,5 & 1,0 & 0 & 0,8 & 15,7 & 32,0 & 37,3 \\
\hline & & -5 & 14 & 6,0 & 3,3 & 1,3 & 12,6 & 0,4 & 0 & 0,9 & 15,7 & 30,9 & 36,9 \\
\hline & & -15 & 15 & 3,7 & 2,7 & 1,3 & 9,6 & 0,4 & 0,2 & 0,7 & 19,1 & 31,3 & 35,0 \\
\hline & \multirow[t]{3}{*}{ Résineux } & Lit. & 16 & 4,0 & 2,3 & 1,4 & 14,8 & 0,3 & 0 & 0,5 & 16,8 & 33,8 & 37,8 \\
\hline & & -5 & 17 & 5,0 & 2,7 & 1,3 & 12,2 & 0,2 & 0,3 & 0,5 & 14,0 & 28,5 & 33,5 \\
\hline & & -15 & 18 & 2,7 & 2,0 & 1,3 & 8,8 & 0,2 & 0,2 & 0,7 & 18,5 & 29,7 & 32,4 \\
\hline \multirow[t]{6}{*}{ Peyrelevade } & \multirow[t]{3}{*}{ Epicéa } & Lit. & 19 & 2,6 & 2,0 & 0,8 & 3,3 & 0 & 0,2 & 1,2 & 21,9 & 27,4 & 30,0 \\
\hline & & -5 & 20 & 7,1 & 4,0 & 1,0 & 1,2 & 0 & 0,3 & 0,9 & 15,1 & 18,5 & 25,6 \\
\hline & & -15 & 21 & 6,8 & 4,0 & 1,2 & 0,8 & 0 & 0,2 & 0,7 & 20,8 & 23,7 & 30,5 \\
\hline & \multirow[t]{3}{*}{ Lande } & Lit. & 22 & 2,7 & 2,0 & 1,3 & 7,5 & 0 & 0 & 0,5 & 20,2 & 29,5 & 32,2 \\
\hline & & -5 & 23 & 7,1 & 4,0 & 1,2 & 5,4 & 0 & 0,2 & 0,8 & 15,1 & 22,7 & 29,8 \\
\hline & & -15 & 24 & 3,9 & 3,0 & 0,8 & 4,0 & 0 & 0,2 & 0,7 & 20,8 & 26,5 & 30,4 \\
\hline \multirow[t]{9}{*}{ Ardennes } & \multirow[t]{3}{*}{ Chêne } & Lit. & 25 & 4,6 & 2,3 & 1,2 & 7,2 & 1,9 & 0,2 & 0,6 & 22,4 & 33,5 & 38,1 \\
\hline & & -5 & 26 & 8,0 & 3,7 & 0,8 & 3,9 & 0,8 & 0,2 & 1,2 & 18,0 & 24,9 & 32,9 \\
\hline & & -15 & 27 & 5,3 & 4,0 & 0,7 & 1,7 & 0,4 & 0,3 & 1,0 & 10,2 & 14,3 & 19,6 \\
\hline & \multirow[t]{3}{*}{ Hêtre } & Lit. & 28 & 3,8 & 1,7 & 0,8 & 10,9 & 2,1 & 0,3 & 0,9 & 19,1 & 34,1 & 37,9 \\
\hline & & -5 & 29 & 6,4 & 3,0 & 1,2 & 11,8 & 2,1 & 0,2 & 1,0 & 14,6 & 30,9 & 37,3 \\
\hline & & -15 & 30 & 5,0 & 3,3 & 1,0 & 2,8 & 0,7 & 0,2 & 0,9 & 12,0 & 17,6 & 22,6 \\
\hline & \multirow[t]{3}{*}{ Epicéa } & Lit. & 31 & 1,4 & 1,0 & 0,9 & 8,6 & 1,0 & 0 & 0,7 & 18,0 & 29,2 & 30,6 \\
\hline & & -5 & 32 & 7,3 & 4,7 & 0,8 & 5,2 & 0,6 & 0 & 0,6 & 14,0 & 21,2 & 28,5 \\
\hline & & -15 & 33 & 6,2 & 4,0 & 1,0 & 1,5 & 0,6 & 0,2 & 0,6 & 9,9 & 13,8 & 20,0 \\
\hline \multicolumn{2}{|c|}{ Vermiculite initiale } & & 34 & 0,2 & 0 & 0,8 & 2,6 & 0 & 0 & 0,6 & 27,2 & 31,2 & 31,4 \\
\hline
\end{tabular}

La dynamique de l'ion Ca est réglée par 3 paramètres :

- La richesse du milieu minéral (appréhendé par le niveau moyen de $\mathrm{Ca}$ sur la $\mathrm{CEC}$ ) opposant nettement Peyrelevade aux 3 autres stations; le type de pédogenèse n'a pas d'influence capitale.

- Le cycle biologique : le milieu acide provoque la désaturation du complexe absorbant en $\mathrm{Ca}$ (en particulier au niveau des litières), mais celui-ci, apporté massivement par les retombées biologiques (VEDY, 1973), tend à s'accumuler en surface (sa bivalence favorise sa compétitivité). Le $\mathrm{Mg}$ peut aussi être apporté par les retombées biologiques.

- L'aptitude d'une essence à recycler le $\mathrm{Ca}$ (toutes choses égales par ailleurs) : le cas de la forêt d'Eu est typique avec le douglas et le hêtre qui montrent une grande facilité de recyclage du $\mathrm{Ca}$ par opposition au groupe épicéa et mélèze. On note d'ailleurs que l'épicéa, d'une manière générale, est l'essence testée qui recycle le plus mal le $\mathrm{Ca}$.

Il ressort donc que les minéraux placés sous les litières seraient soumis à un régime complexe à la fois lessivant, sous l'influence directe des acides organiques, et " enrichissant », sous l'influence de cations libérés par la minéralisation des litières avec un scénario voisin pour tous les milieux. L'état du complexe absorbant des minéraux issus des horizons plus profonds discrimine mieux le type de pédogenèse : le milieu podzolique conserve toujours à $-15 \mathrm{~cm}$ une agressivité importante (très net à Cires-lès-Mello) par opposition aux sols bruns acides; le comportement du $\mathrm{Mg}$ traduit bien ces phénomènes.

Au niveau espèce, la spécificité de l'épicéa se traduit sous les litières à la fois par une saturation en cations basiques et une acidité d'échange faibles, et, sous l'horizon $A_{11}$, par une saturation (relative) faible en cations basiques.

Les critères CEC et saturation cationique opèrent donc déjà, après 18 mois d'expérimentation, une certaine discrimination en fonction des stations des essences et des horizons, l'effet essence se faisant sentir surtout en surface, alors que c'est l'inverse pour l'effet station. Il est également remarquable d'observer comment une espèce (en l'occurrence l'épicéa) produit la même évolution relative de la CEC, en fonction des stations. 


\section{Les transformations minéralogiques}

La transformation du minéral placé en sachet, telle qu'elle peut être caractérisée par diffraction des rayons $\mathrm{X}$, a été assez nette, ceci malgré la faible durée de l'expérience.

$\mathrm{Au}$ plan de la description, la déstabilisation du minéral initial porte d'une manière évidente sur la vermiculite et/ou sur l'interstratifié, il n'est cependant pas exclu que la partie mica (pic à $10 \AA$ ) subisse également une évolution par ouverture des feuillets, même si celle-ci reste difficile à observer.

Divers types d'évolutions se manifestent de manière nette et nous avons tenter de les relier à l'évolution physicochimique observée, concernant en particulier la CEC. Les résultats sont reportés sur la figure 4 .

Au total, les transformations du minéral (CEC + évolution minéralogique) donnent 6 cas de figures.

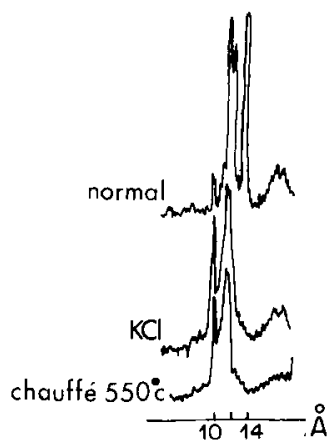

Vermiculite initiale

Situation des exemples

Eu (NORMANDIE) : 1 _ 2b_ 5

Cirés lès Mello (B. PARISIEN): 20.3

Peyrelevade (MASSIF CENTRAL): 4

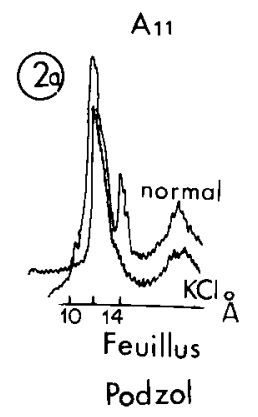

(2b)

meq $/ 100 \mathrm{~g} \_$Sol brunacide
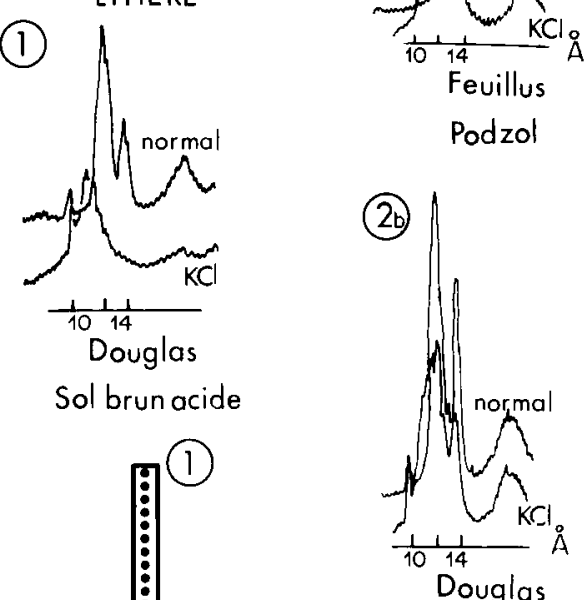

Sol brun acide

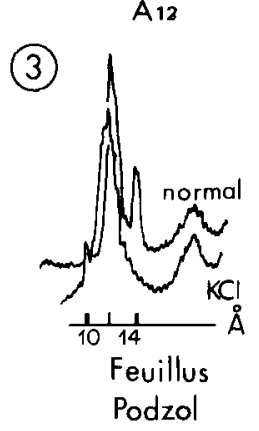

(3)

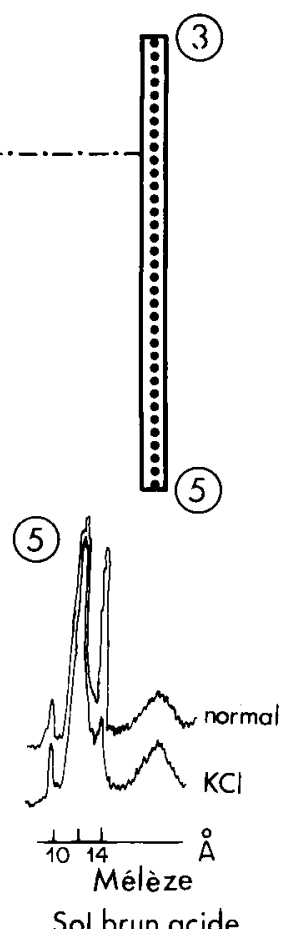

$A_{12}$ 
1) Sous les litières, on constate que la CEC est stable ou augmente plus ou moins fortement : la réponse du minéral se traduit par une diminution du pic à $14 \AA$ de la vermiculite (en saturation issue du contact avec le sol) et un transfert du pic à $12 \AA$ de l'interstratifié vers $11,4 \AA$ après traitement au $\mathrm{KCl} \mathrm{N}$ (cas $n^{\circ} 1$ ).

2) Dans l'horizon intermédiaire $A_{11}(-5 \mathrm{~cm})$ et dans l'horizon $A_{12}(-15 \mathrm{~cm})$, plusieurs possibilités se présentent :

- la CEC augmente et l'on peut observer 2 variantes :

a) une évolution similaire à celle des litières (cas $n^{\circ} 2 \mathrm{a}$ et 3 ),

b) une évolution caractérisée par une certaine déstabilisation de l'interstratifié (déplacement partiel du pic à $12 \AA$ après saturation $\mathrm{KCl}$ ) couplée à une évolution du pic à $14 \AA$ qui se conserve partiellement après saturation $\mathrm{KCl}$ (cas $\mathrm{n}^{\circ} 2 \mathrm{~b}$ ) ;

- la CEC diminue : le pic à $12 \AA$ de l'interstratifié n'est apparemment pas affecté, le pic à $14 \AA$ de la vermiculite ne se referme que partiellement après la saturation potassique (cas $n^{\circ} 4$ et 5 ).

$\mathrm{Au}$ total, ces 6 cas de figures se regroupent en 3 types d'évolution comme suit : type 1 (cas $n^{\circ} 1,2 a$ et 3 ), type 2 (cas 2 b), type 3 (cas 4 et 5 ).

\section{E. Essai d'interprétation de la relation évolution minéralogique-évolution physicochimique}

Nous traitons séparément les évolutions des séquences du minéral interstratifié mica-chlorite et celle de la vermiculite. Ce matériau mixte permet de plus une interprétation de l'évolution relative des séquences minérales. La transformation de l'interstratifié micachlorite vers un interstratifié de type mica-vermiculite se caractérise par une libération des sites d'échanges initialement saturés par du Mg non échangeable (type brucite). L'aspect qualitatif de cette évolution, globalement proportionnelle à l'augmentation de CEC est assez facile à caractériser (cas $n^{\circ} 1$ ).

$$
\text { L'étude du rapport } \frac{\text { Hauteur du pic à } 10 \AA}{\text { Hauteur du pic à } 12 \AA} \text { (en }
$$

saturation normale) qui permettrait une approche semi-quantitative ne donne aucun résultat puisqu'au total l'interstratifié se conserve même si une évolution qualitative se produit.

La transformation de la vermiculite se traduit :

- par une diminution du pic à $14 \AA$ (en saturation cationique issue du contact avec le sol). Cette diminution caractérise une certaine fermeture du minéral, vraisemblablement due à des cations non hydratés tels $\mathrm{K}^{+}$ou $\mathrm{NH}_{4}^{+}$provenant du milieu. Ce processus ne libère en aucun cas des sites échangeables susceptibles d'expliquer l'augmentation de CEC ; il est même probable que ces cations sont « rétrogradés » donc exclus du pool des ions échangeables. On observe une relation linéaire significative $(r=0,601 \mathrm{n}=36)$ entre augmentation de $\mathrm{CEC}$ et diminution du rapport Hauteur du pic à $12 \AA$

Hauteur du pic à $14 \AA$

(en saturation cationique issue du contact avec le sol) ; cette relation traduit le fait que le flux de cations efficaces pour la fermeture des feuillets est maximum sous les litières (cas $n^{\circ} 1$ ) ;
- par une persistance partielle du pic à $14 \AA$ après saturation $\mathrm{KCl}$ traduisant une agradation de la vermiculite stabilisée par un cation hydroxylé non échangeable.

Cette évolution se caractérise par une diminution de la CEC, qui résulte à la fois d'une stabilité apparente de l'interstratifié et d'un blocage d'une partie des sites échangeables de la vermiculite (cas $n^{\text {os }} 4$ et 5 ). Il existe cependant un groupe d'échantillons qui présente à la fois l'agradation de la vermiculite et la déstabilisation partielle de l'interstratifié avec comme résultante une légère augmentation de la $\mathrm{CEC}$ (cas $\mathrm{n}^{\circ} 2 \mathrm{~b}$ ).

$\mathrm{Au}$ total, la confrontation des résultats concernant l'évolution physicochimique et l'évolution minéralogique est satisfaisante. Il resterait cependant à expliquer le cas particulier du groupe d'échantillons qui présente 2 types d'évolution minéralogique pour une même évolution de la CEC.

\section{F. Caractérisation de I'agradation}

Le test MEHRA-JACKSON (1960) permet de préciser la nature de l'élément responsable de l'agradation.

Les résultats moyens sont groupés dans le tableau 3 ; fer et silice ne montrent pas de grandes variations entre horizons ; seul l'aluminium varie d'une manière significative. Il existe de plus une relation entre $\mathrm{Al}$ libre et CEC ( $\mathrm{r}=-0,709 \mathrm{n}=33$ ) montrant le rôle de cet élément dans le processus d'évolution de la vermiculite (fig. 5).

Les diffractogrammes effectués après le test MEHRA-JACKSON et une saturation potassique montrent le retour à un comportement normal de la vermiculite.

Il s'agit donc là d'une « agradation alumineuse », signalée par de nombreux auteurs parmi lesquels JACKSON (1962), RICH (1968), RAZZAGHE-KARIMI (1976), mais le plus souvent obtenue au laboratoire ; les résultats «quasi expérimentaux » obtenus ici in situ permettent de montrer qu'il s'agit là d'un phénomène généralisé et rapide pour les sols acides.

Dans le détail, le nuage de points de la figure 5 se divise en 2 parties séparées par l'axe $\mathrm{AB}$ : d'une part, les minéraux issus des litières et $\mathrm{du}$ podzol de Cireslès-Mello, d'autre part, les horizons hémiorganiques des sols. Le podzol humifère de Peyrelevade a une position intermédiaire.

Le type de pédogenèse influe donc nettement sur la dynamique de l'Al dont le confinement ne peut se réaliser que dans les horizons moyens des sols brunifiés

TABLEAU 3

Les éléments libres (résultats exprimés en $\mathrm{g} \%$ oo).

Free oxides (data in g per 1000 parts of air dried mineral).

\begin{tabular}{ccccccc}
\hline & \multicolumn{2}{c}{$\mathrm{Fe}$} & \multicolumn{2}{c}{$\mathrm{Si}$} & \multicolumn{2}{c}{$\mathrm{Al}$} \\
\hline & & & & & & \\
$\mathrm{m}$ & $\sigma$ & $\mathrm{m}$ & $\sigma$ & $\mathrm{m}$ & $\sigma$ \\
$\mathrm{Lit}$ & 0,48 & 0,09 & 0,91 & 0,28 & 0,18 & 0,06 \\
$\mathrm{~A}_{11}$ & 0,51 & 0,12 & 0,79 & 0,32 & 0,44 & 0,13 \\
$\mathrm{~A}_{12}$ & 0,56 & 0,07 & 0,60 & 0,27 & 0,53 & 0,23 \\
\hline
\end{tabular}

$\mathrm{m}=$ moyenne $; \sigma=$ écart type. 


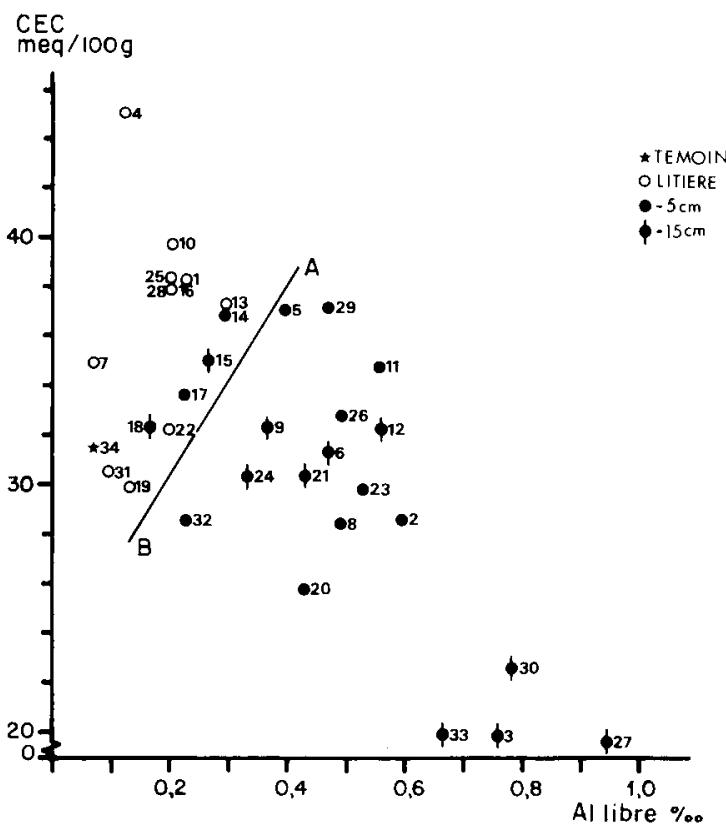

Figure 5

Relation CEC et aluminium libre (MJ).

Interrelation between $C E C$ and aluminium extracted by MehraJackson method.

(Eu et Monthermé) : c'est dans ces horizons que le blocage de la CEC est le plus net. L'agradation alumineuse n'existe que si le minéral est expansible ; SADIO (1982) n'observe pas d'agradation d'une parabiotite malgré le confinement d'Al dans les horizons profonds de sols vosgiens.

\section{G. Essai de synthèse d'interprétation du compor- tement du minéral dans l'expérimentation}

L'étude de la transformation du "minéral-test » montre que des modifications complexes touchant la chimie, la physico-chimie et la minéralogie sont inter- venues, ceci en une période de temps relativement courte.

On obtient un schéma explicatif cohérent des divers résultats obtenus en intégrant les 3 paramètres : CEC, évolution minéralogique et taux d'Al libre, comme le montre clairement le tableau 4 (un seul échantillon n'entrant pas totalement dans le schéma).

On voit l'apport du paramètre $\mathrm{Al}$ libre (MJ), selon que l'agradation existe ou non comme élément explicatif de l'évolution du minéral, en particulier au niveau du groupe d'échantillons pour lesquels une même valeur de la CEC correspond à 2 types de transformations minéralogiques. (Ces échantillons sont ceux issus des horizons $A_{11}$ et $A_{12}$ des sols pour lesquels la CEC se maintient ou augmente.) La valeur de l'Al libre discrimine les milieux brunifiés (Eu et Monthermé) et podzolisés (Cires). Le seuil à partir duquel on peut avoir l'agradation par $\mathrm{Al}$ du minéral-test a été fixé empiriquement à 0,33 p. 1000 .

Il .est important de préciser que les 3 paramètres pris en compte sont complémentaires et permettent de rendre compte de l'évolution du minéral test, même dans le cas où l'un des paramètres semble ne pas être modifié. Le cas de la stabilité apparente de la CEC dans un certain nombre de situations en est un bon exemple. Cette même valeur de CEC avant et après traitement correspond à des saturations cationiques très différentes (cf. III.C) traduisant elles-mêmes des processus évolutifs différents.

\section{CONCLUSION}

Il existe peu de méthodes pour caractériser le fonctionnement biogéochimique actuel des sols. Les études portant sur les eaux apparaissent particulièrement complexes à réaliser et doivent prendre en compte toutes les variations du fonctionnement susceptibles de se produire au cours de l'année (BONNE et al., 1982).

TABLEAU 4

Schéma de l'évolution de la "vermiculite du Kenva » après 18 mois de contact dans diverses conditions stationnelles (sol $\times$ essence). Diagram showing of the evolution of « vermiculite from Kenya " after 18 months of contact with soils in different ecosystems (soil $\times$ species).

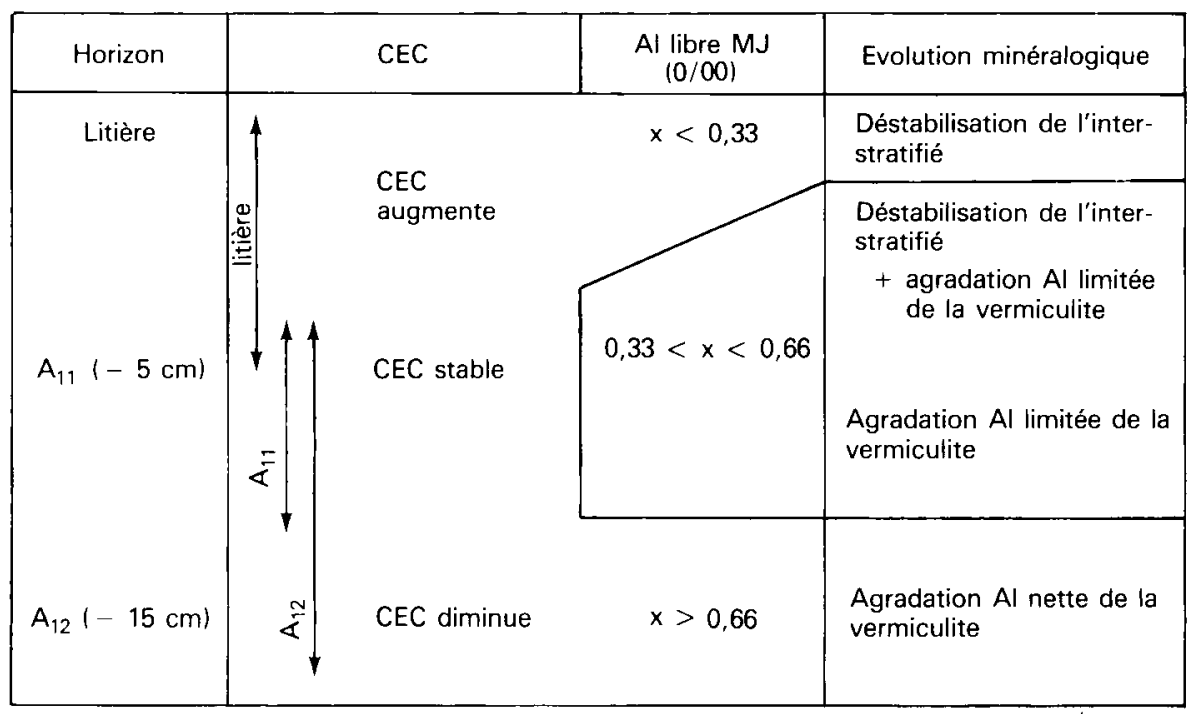


D'un autre côté, les études minéralogiques basées sur des bilans (LELONG \& SOUCHIER, 1979 ; NYS, 1981) ou sur l'évolution de la fraction " argile " (ROBERT et al., 1980) peuvent révéler un processus dominant, mais elles intègrent en général, toute l'histoire et la genèse du sol. La caractérisation des éléments libres et celle du complexe d'échange des minéraux argileux du sol (EsPiaU, 1978 ; EsPIAU \& PEDRO, 1980) apportent déjà des éléments permettant de caractériser les changements intervenus au cours de l'évolution pédologique récente. Il semble que la méthode d'introduction d'un « minéral test » dans un sol soit à ce sujet particulièrement intéressante et prometteuse pour l'avenir.

Le choix du minéral est déterminant. Sur ce point, les premières expériences menées par SADIO (1982) ont montré l'intérêt de cette méthode, mais aussi les limites qui peuvent résulter du choix de minéraux très stables (illites dioctaédriques). Il est donc nécessaire d'utiliser des minéraux sensibles (trioctaédriques à forte $\mathrm{CEC}$ ) susceptibles d'évoluer rapidement in situ.

Le matériau utilisé ici présente une $C E C$ relativement modeste (31 meq/100 g), mais possède l'avantage de juxtaposer dans la même particule plusieurs minéraux susceptibles d'évoluer rapidement, ce qui s'est avéré intéressant dans l'interprétation des résultats. Ce matériau est un minéral complexe présentant une alternance de séquences monominérales (mica et vermiculite) et des séquences interstratifiées régulières (mica-chlorite).

Il était important de vérifier si le minéral peut évoluer rapidement et si les différents résultats présentés sont cohérents et permettent de préciser le rôle des essences sur l'orientation des évolutions au sein des différents milieux pédologiques testés.

La comparaison du minéral avant et après expérimentation montre que des transformations importantes ont eu lieu, après une courte période de 18 mois de contact ; elles ont porté sur les éléments les plus accessibles : éléments échangeables et éléments libres. Ces transformations se reflètent au niveau de l'évolution minéralogique caractérisée par diffraction des rayons $\mathrm{X}$.
La prise en compte des paramètres CEC, saturation cationique, transformation minéralogique et éléments libres fournit une norme d'interprétation cohérente.

L'étude globale à l'aide de relations statistiques nous a permis de mettre en évidence les processus d'évolution.

- En ce qui concerne les cations échangeables, la relation négative qui lie l'aluminium et le magnésium, traduisant des processus géochimiques divergents, est tout à fait confirmée par l'évolution minéralogique des échantillons ayant séjourné dans les litières et dans les sols. De plus, ces évolutions entrent parfaitement dans le schéma théorique établi par ROBERT (1970) et ROBERT et al. (1979).

- La relation CEC-Al libre (MJ) montre le rôle de cet élément dans la transformation minéralogique de la vermiculite. L'aluminisation des couches interfoliaires peut donc être un phénomène rapide.

L'interprétation détaillée des résultats permet à l'intérieur des relations générales de relier la variabilité observée à un type de milieu, de pédogenèse, ou encore à une espèce testée.

Une certaine discrimination se manifeste, avec globalement un effet essence dominant dans les échantillons issus des litières et des horizons $A_{11}$. L'effet station l'emporte dès $15 \mathrm{~cm}$ de profondeur, avec une distinction nette entre la série podzolique et les sols bruns acides.

Il est évident que nous n'avons répondu actuellement qu'à une partie de la question posée, mais ces premiers résultats sont très positifs pour la poursuite de cette voie expérimentale qui permet d'avoir rapidement des informations sur l'orientation des mécanismes physico-chimiques (dissolution, échange des cations) intéressant le fonctionnement réel des sols. On peut donc penser que cette approche pourrait être utilisée pour d'autres problèmes actuels de science du sol : pollution, irrigation par les eaux saumâtres...

Recu le 12 août 1983. Accepté le 4 octobre 1984.

\section{RÉFÉRENCES BIBLIOGRAPHIQUES}

Berthelin J., Sadio S., Guillet B., Rouiller J., 1983. Altération expérimentale in situ de minéraux argileux di et trioctaédriques dans un podzol et un sol brun acide. Pétrologie des altérations et des sols. Coll. int., CNRS, Paris (sous presse).

Bonne M., Andreux F., Vedy J. C., Souchier B., 1982. Etude hydrochimique saisonnière dans trois sols acides : composition des eaux gravitaires et des solutions extraites à pF 4,4. Sci. Sol, 4, 275292.

Bonneau M., Lacaze J. F., Lelong F., Levy G., Nys C., Souchier B., Brethes A., 1977. Modification de fertilité des sols sous boisements artificiels de résineux purs. C. R. fin d'étude DGRST, 1, vol. ronéot., 88 p., Doc. int. CNRF.

Compte rendu ASP Pirfo N ${ }^{\circ} 4,1982$. Conséquences de la monoculture des résineux et alternatives possibles. Rapport CNRS, 1 vol., 67 p. + annexes.

Espiau P., 1978. Etude d'une série de sols de l'étage montagnard de Corse ; contribution de la minéralogie des argiles et de l'acidité d'échange à l'interprétation de la pédogenèse. Sci. Sol, 3, 167-183.
Espiau P., Pedro G., 1980. Caractérisation du complexe d'échange des sols acides. Le taux d'acidité d'échange et sa signification pédogénétique sous climat tempéré. Ann. Agron., 31, 4, 363-383.

Herbaults J., Debuyle, 1981. The relation between spruce monoculture and incipient podzolisation in ochreous brown earths of the Belgian Ardennes. Plant Soil, 59, 33-49.

Jackson M. L., 1962. Interlayering of expansible layer silicates in soils by chemical weathering. Clays Clay Miner., 29-46.

Jeanroy E., 1974. Analyse totale par spectrophotométrie d'absorption atomique, des roches, sols minéraux, ciments, après fusion au métaborate de strontium. Analusis, 2, 703-712.

Lelong F., Souchier B., 1979. Les bilans d'altération dans les sols, méthodes, résultats, perspectives. Sci. Sol, 2, 3, 267-279.

Mehra O. P., Jackson M. L., 1960. Iron oxide removal from soils and clays by a dithionite-citrate system buffered with sodium bicarbonate. Clays Clay Miner., 7, 317-327.

Noirfalise A., Vanesse R., 1975. Conséquences de la monoculture 
des conifères pour la conservation des sols et le bilan hydrologique. Association espaces verts Bruxelles, 1-25.

Nys C., 1975. Un podzol humo-ferrugineux humifère sur le granite de Millevaches. Sci. Sol, 207-211.

Nys C., 1981a. Evolution des sols sous différentes essences forestières: exemples de la forêt d'Eu (Normandie) et de Cires-lès-Mello (Bassin parisien). Doc. Int. CNRF.

Nys C., 1981b. Modification des caractéristiques physico-chimiques d'un sol brun acide des Ardennes primaires par la monoculture d'épicea. Ann. Sci. For., 38, 2, 237-258.

Nys C., Ranger D., Ranger J., 1983. Etude comparative des deux écosystèmes forestiers feuillus et résineux des Ardennes primaires françaises. III. Minéralomasse et cycle biologique. Ann. Sci. For., 40, 1, 41-66.

Razzaghe-Kamiri M. H., 1976. Contribution à l'étude expérimentale des phénomènes d'altération en milieu organique acide. Application à l'évolution des micas et phyllosilicates 2/1. Thèse Doct. Etat, Univ. Paris VI, 222 p.

Rich C. I., 1968. Hydroxy-interlayers in expansible layer silicates. Clays Clay Miner., 16, 15-30.
Robert M., 1970. Etude expérimentale de la désagrégation du gra nite et de l'évolution des micas. Thèse de Doct. Etat. Univ. Paris, 194 p. in Ann. Agron., 1970, 21 (6), $777-817$ et Ann. Agron., 1971, 22 (1), 43-93.

Robert M., Razzaghe-Karimi M. H., Vicente M. A., Veneau G., 1979. Rôle du facteur biochimique dans l'altération des minéraux silicatés. Sci. Sol, 2, 3, 153-174.

Robert M., Cabidoche Y. M., Berrier J., 1980. Pédogenèse et minéralogie des sols de haute montagne cristalline. Sci. Sol, 4, 313-336.

Rouiller J., Guillet B., Bruckert S., 1980. Cations acides échangeables et acidités de surface. Approche analytique et incidences pédogénétiques. Sci. Sol, 2, 161-178.

Sadio S., 1982. Altération expérimentale des phyllosilicates - tests sous végétations forestières acidifiantes. Thèse Doct., Ing., Nancy I, $95 \mathrm{p}$.

Vedy J. C., 1973. Relations entre le cycle biogéochimique des cations et l'humification en milieu acide. Thèse Doct. Etat, Univ. Nancy I, $116 \mathrm{p}$. 